\title{
Estrategia de aprendizaje en la formación ambiental con estudiantes de las Ciencias Agrarias
}

(C) Copyright 2021. Universidad Nacional Autónoma de Nicaragua, Managua (UNAN-Managua) Todos los derechos reservados

\section{Learning strategy in environmental training with agricultural science students}

Indiana Ramona Montoya Dompé

Docente del Departamento de Ciencias Tecnología y

Salud, FAREM-Chontales

https://orcid.org/0000-0003-4832-1121

imontoya@unan.edu.ni

Fecha de recibido: 13/04/2021

\section{Resumen}

En la actualidad, los procesos de aprendizaje de las ciencias agrarias deben volcar la mirada a fortalecer la temática ambiental y protección de los medios de vida, a transformar la ciencia y la tecnología a favor de una educación de calidad, orientada a fortalecer la temática ambiental, ya que permite una combinación extraordinaria entre los saberes y los quehaceres en el campo. Se ha desarrollado un estudio exploratorio, basándose desde un enfoque cualitativo, con el afán de comprender las valoraciones percibidas por los actores que han participado de estas experiencias. En palabras de los estudiantes "consideran que los conocimientos adquiridos nos benefician a saber trabajar la tierra sin degradarla" uno de los hitos de mayor relevancia en esta experiencia, es haber roto esquemas de la relación familia-hijos, puesto que los padres, al dejar a sus hijos realizar estos experimentos en sus propios lugares, consideran un paso importante en la vida de los jóvenes, que van cambiando su propio entorno. Estas acciones permiten armonizar actitudes, esquemas mentales y prácticas productivas que estén a la par de los desafíos del futuro.

\section{Narciso Lenin Duarte Acevedo}

Docente del Departamento de Ciencias Tecnología y

Salud, FAREM-Chontales

https://orcid.org/0000-0002-9336-4086

nduarte@unan.edu.ni

\section{Palabras claves}

Experiencias significativas, comunidad, educación rural.

\section{Summary}

At present, the learning processes of agricultural sciences must turn their gaze to strengthen the environmental issue and protection of livelihoods, to transform science and technology in favor of quality education, aimed at strengthening environmental issues, since it allows an extraordinary combination of knowledge and tasks in the country side. An exploratory study has been developed, for which a qualitative approach was taken, with the aim of understanding the valuations perceived by the actors who have participated in these experiences. In the words of the students, "they consider that the knowledge acquired benefits us to know how to work the land without degrading it" one of the most relevant milestones in this experience is to have broken schemes of the family-child relationship, since parents leave their children carry out these experiments in their own 
places, it is an important step in the lives of young people, who are changing their own environment. These actions make it possible to harmonize attitudes, mental schemes and productive practices that are on par with the challenges of the future.

\section{Keywords}

Significant experiences, community, rural education.

\section{Introducción}

El presente artículo pretende compartir experiencias educativas en la enseñanza y el aprendizaje del componente ambiental en estudiantes de las disciplinas de las Ciencias Agrarias, a partir de la reflexión de los errores y aciertos de muchos años de labor docente, ya que para profesionales de áreas especializadas entrar en el universo educativo es una aventura y un desafío, especialmente a las puertas de las nuevas demandas en la Educación Superior. En donde se pide a la universidad que sea el motor de los cambios y que forme a los estudiantes para que transformen y construyan una sociedad mejor (Moore, et al. 2018) y por las propias complejidades del proceso de aprendizaje, elemento esencial de la vida de los seres humanos.

Como afirman Melgary Donolo (2011), se hace necesario ofrecer múltiples perspectivas para enseñar y aprender diversos enfoques y prácticas para comprender el mundo recuperando no solo la perspectiva del docente, sino también la de otros actores, como los alumnos, sus familias, el ambiente, los medios de comunicación, la comunidad, otras instituciones educativas; al mismo tiempo que incorpora propuestas que combinen el aprender con otros, en distintos lugares y con diversos recursos, ajustados a la cultura, a los recursos locales, a las personalidades, a las experiencias, historias $\mathrm{y}$ potencialidades individuales y colectivas de los estudiantes.

Este proceso para el docente implica una participación activa, una motivación constante mediante la acciónreflexión-acción que permita crear condiciones para un aprendizaje creativo, con una continua experimentación y el diseño de estrategias que motiven y ejerciten al estudiante a aprender a aprender desde un vínculo directo con la vida, es ahí donde el correcto diseño y uso de las escuelas o tiempo en el campo del aprendizaje de asignaturas ambientales se vuelven más que una herramienta pedagógica, sino el corazón del propio proceso de aprendizaje (López, 2017).

En ese sentido, el progreso científico pone de manifiesto avances en la solución del problema alimentario actual, en donde datos de la Organización de las Naciones Unidas para la Alimentación y la Agricultura demostró el número de personas que sufren hambre en el mundo, ascendiendo a 690 millones en 2019. Es ahí donde se hace necesario asociar la educación de los futuros profesionales de las ciencias agrarias con procesos de productividad y calidad que permitan seguir avanzando en el desarrollo de la nación mediante un marco de sustentabilidad de los recursos empleados, pues la alimentación es la base fundamental para la sobrevivencia de la humanidad.

El componente ambiental aplicado al campo agropecuario tiene aplicaciones directas en los contenidos diseñados en las universidades, en ese sentido la UNAN-Managua, al ser una universidad pública, ha considerado que los escenarios en donde se desarrollen estos procesos sean factibles, es decir, ocupando los espacios y oportunidades con los que cuenta la universidad. Con ello se respalda que los estudiantes tengan una apropiación de las problemáticas vividas en el mundo rural y la aplicación de conocimientos que, de manera independiente, puedan cambiar su entorno a través de los potenciales desarrollados; ya que los jóvenes provienen de diversos contextos, especialmente de la pobreza. Es por ello que se ve a los jóvenes como la principal arma de empoderamiento ante este flagelo.

Puesto que la humanidad ha entrado en una nueva fase de su historia con la evolución cada vez más rápida en ciencia y tecnología, y por consiguiente, puedan beneficiarnos de manera enmancipatoria, justa y sostenible, es preciso comprender y gestionar las oportunidades y los riesgos. Posibilitarlos debería ser el propósito fundamental de la educación y el aprendizaje en el SXXI. .

La novedad de la educación 2030 es que se centra en el afianzamiento y ampliación del acceso, de la inclusión y la equidad, de la calidad y los resultados del aprendizaje en todos los niveles, como parte de un enfoque de aprendizaje a lo largo de la vida (UNESCO, 2015b). 
Para Ávila \& Cortés (2017) para promover el desarrollo de competencias en los universitarios, se hace necesario impulsar el pensamiento crítico, que conduce a incorporar elementos como la reflexión, recreación y reinversión del aprendizaje, la participación que el empoderamiento de los actores sobre su práctica. Asumir de manera consciente sus acciones, además de ser corresponsables de las relaciones con otros, conduce a experiencias solidarias y de crecimiento mutuo.

Con este escrito, se pretende impulsar a los docentes a salir de la zona de confort, a crear procesos propios, a emocionarse por crear espacios de reflexión sobre los resultados obtenidos de las acciones y tomas de decisiones acerca de cómo se pueden agilizar los cambios en la era del conocimiento.

\section{Perspectiva del docente ante lo nuevo}

Cuando se concibe una clase, el miedo al rechazo de las metodologías implementadas implica un riesgo a asumir. Es importante ampliar los horizontes y saber valorar los momentos propios de aprendizaje y los momentos de los demás. Es como sentirte más que feliz.

El miedo es eso que por un minuto te pone en pausa, ese minuto se eterniza al pensar si podés hacerlo, si es correcta la propuesta o no. Ese minuto se transporta al otro lado del escritorio intentando descubrir que movería las emociones de los estudiantes -no omitiendo que cada generación es diferente, parecida más a sus tiempos que a las actividades y dirección de vida que tienen los jóvenes- ¿que los motivaría a hacer "algo"?, puesto que constantemente se observan jóvenes desmotivados y la idea es darles esa pulsación inicial. La motivación constituye el motor más poderoso para desarrollar y mantener un aprendizaje. Bartolomé (2017), la entiende como un estado de energía, excitación o intensidad emocional, activa, dirige y mantiene una conducta hacia un objetivo concreto.

Es difícil conjugar todos esos elementos al pensar en una clase que signifique mucho para ellos, las innovaciones en el campo educativo no suceden de un día para el otro sino que requieren de inversiones importantes de los sujetos implicados, quienes deben invertir tiempo, energía y pasión en la construcción de nuevos contextos para aprender y enseñar.
Una vez superado ese minuto de miedo, se prosigue a la planificación de actividades que motiven un aprendizaje significativo -que es un acto más bien instintivo que estructurado con todos los elementos pedagógicos. Cada clase se vive por instantes, en donde el profesor prácticamente es un espectador que, de alguna manera, guía simplificadamente hacia la consecución de un objetivo planeado. Como dice Paulo Freire (1981) "la totalidad del conocimiento, a partir de una temática específica, se va extendiendo a medida que esa misma es problematizada en sus diversas posibilidades. En este sentido, la problematización "[...] es la reflexión que alguien ejerce sobre un contenido, resultado de una acción, o sobre la propia acción".

En pocas palabras, este es el aporte que se extrae desde el interior hacia el exterior, pues busca una respuesta propia para construir y reconstruir la vida en la educación de manera constante, que es consistente con el nuevo modelo educativo de Nicaragua, basado en la utilización del conocimiento como materia prima para crear valor con él, convirtiéndolo en fuente de desarrollo personal y profesional. En términos de desarrollo, se quiere de los principales actores aprendan a interrelacionar los saberes, y para ello deben conocerse a sí mismos, aprender y convertir la realidad.

\section{Disposiciones metodológicas}

Se ha desarrollado un estudio exploratorio, para lo que se tuvo un enfoque cualitativo con el afán de comprender las valoraciones percibidas por los actores que han participado de estas experiencias. En total, han participado 47 estudiantes de las carreras de Ingeniería Agronómica y Desarrollo Rural Sostenible respectivamente y dos docentes.

Se utilizó un cuestionario con 7 preguntas abiertas y la narrativa para el docente. Las preguntas incluyeron aspectos como: la calidad de los contenidos, los materiales de estudio facilitados, la relación entre los contenidos y valoraciones generales de la experiencia. Los docentes analizaron desde sus vivencias y emociones experimentadas en los diferentes momentos de la propuesta desde la preparación de la clase hasta la evaluación. 


\section{Descripción de las experiencias}

Las experiencias se realizaron en las clases de Edafología I, Manejo Forestal y Sistemas de Producción Animal, con el entendido que se preparó una metodología en donde se practicó la inclusión de las competencias para el SXXI de manera práctica aplicando el aprendizaje colaborativo ajustados a las normas que tiene la UNAN-Managua (percibiendo) el toque innovador.

La Agronomía como carrera está ligada al mundo rural, y las asignaturas están interrelacionadas entre sí, es decir la articulación entre el medio ambiente, el suelo y los animales, como un todo que dependen como pilares únicos en su entorno. Contienen elementos que son la base para cimentar el futuro, las prácticas agropecuarias inadecuadas a lo largo del tiempo han traído consigo un desbalance entre los principios ecológicos naturales y la convivencia que tiene el ser humano con su medio.

La relación: humano, suelo, bosques y producción, tiene que buscar la armonización de actitudes, esquemas mentales y prácticas productivas que estén a la par de los desafíos de futuro, teniendo siempre presente la calidad y gratuidad de una educación que impulse a la creación de un proyecto vital que fortalezca la dinámica del país, es así, que la formación de jóvenes en el sector rural como una forma de capitalizar las zonas rurales del país donde se ve necesario el recurso humano, se pueda acceder de una manera comprometida en su formación profesional.

En general, la UNAN-Managua aporta al docente un Maletín Didáctico que contiene el Programa de la Asignatura, formatos de planificación semestral, semanal y diario; así como formatos evaluativos. Los procedimientos para la planificación son sencillos: descriptor de la unidad a desarrollar, los objetivos conceptuales, procedimentales y actitudinales; los contenidos a tratar, estrategias de enseñanza aprendizaje y formas de evaluación.

En el plan diario de clase, se toma en cuenta los objetivos del tema a tratar, las actividades (divididas en tres momentos: iniciales, de desarrollo y finales) y la evaluación. También se interpusieron desafíos intelectuales que se ajusten a las capacidades de los estudiantes y darles un empujón, ya que una de las partes más importantes es activar "motivación" a los estudiantes y cada vez aumentar sus responsabilidades.
La UNAN-Managua también promueve un programa especial denominado Universidad en el Campo, que utiliza la Metodología Escuela Nueva. Esta consiste en que el interés es en el educando y en el desarrollo de sus capacidades; lo reconoce como sujeto activo de la enseñanza y, por lo tanto, el estudiante posee el papel principal en el aprendizaje. El otro elemento que identifica esta tendencia pedagógica es que la educación se considera como un proceso social que asegura su propio desarrollo.

Según la Fundación Escuela Nueva y su directora Vicky Colbert Arboleda, la estructura metodológica de las guías de aprendizaje comprende cuatro factores básicos: en primer lugar el seguimiento de los procesos lógicos de aprendizaje, la integración de procesos y contenidos, comprensión de un conjunto de actividades que fomentan la interacción social y con el entorno y promoción de la evaluación formativa y la retroalimentación permanentes. (Zapata, 2014)

En la implementación de la experiencia se ha basado en mantener activos a los estudiantes con interés especial en lecturas, clases demostrativas y laboratorios de campo diseñadas, de tal manera, a encaminar u obtener conocimientos aplicados con un alto porcentaje de esfuerzo en el trabajo final, donde deben combinar todo lo aprendido desde la teoría a la práctica, devolviendo conocimientos a productores del campo a través de la Extensión Universitaria; para lo cual se evidencia en videos y fotografías que ilustran los resultados de esta etapa de finalización.

Muchas veces los docentes creemos inspirarnos tanto y no logramos transmitir realmente lo que buscamos; ahí es donde el estudiante encuentra su primera barrera, las herramientas (documentos) que se le facilitan al estudiante se vuelve muy efímero, este a su vez lo desecha de inmediato, no muestra interés, se le hace aburrido.

No obstante, el poder articular una herramienta creada de forma adecuada, sencilla y asimilable que logre unir el aprender con el hacer de forma inmediata, facilita la motivación, pero... ¿cómo hacemos esto? Para muchos, la docencia a nivel superior se debe centrar en abrir la mentalidad del alumno, que resuelva problemáticas reales en un aula de clases o bien en campo, directamente al momento que se presenta... pero... ¿Realmente es así?, se busca eso, pero la experiencia acumulada indica que se puede llegar a tener mucho éxito siempre y cuando se tengan los medios adecuados. 
La hora interesante para los estudiantes es la práctica, ya sea para procesamiento de muestras, identificación de árboles o elaboración de alternativas de alimentación de animales donde se utilizan métodos de campo (métodos fáciles de utilizar) y los laboratorios de campo en donde siguen un instructivo o una guía de aprendizaje. Al estudiante se le despierta la curiosidad, puesto que aprenden una destreza y demuestran sus conocimientos al finalizar estos procesos, ya que anotan los datos obtenidos y proceden a elaborar un informe que exponen al final de la clase, que contiene un análisis completo de lo abordado.

Estas habilidades (planificación, control y valoración) no siempre son insertadas en la actividad de aprendizaje como parte de los procedimientos a adquirir por elalumno, sin embargo, su inclusión se justifica si tenemos en cuenta que son precisamente muchos de estos procedimientos los que se ponen en marcha cuando el sujeto enfrenta las diferentes tareas y problemas, los cuales le exigen orientarse, analizar las condiciones, planificar como llevarlas a cabo, buscardiferentes alternativas de solución, controlar y evaluar el cumplimiento de los objetivos que le permitan hacer reajustes y anticipar las nuevas acciones a realizar, es decir, autorregular su actividad.

Se han obtenido resultados que enriquecen los aprendizajes de los estudiantes como del profesor, se observó que ellos se esmeran por hacer cada paso de la manera correcta y el maestro aprende las maneras en como los estudiantes se organizan y ejecutan su plan de actividades de manera autónoma, cumpliendo los tiempos establecidos.

"las clases son importantes para nuestra formación como futuros ingenieros, porque debemos estar capacitados para cualquier circunstancia que se presente en campo". (V. Villegas, comunicación personal)

Las clases desarrolladas han tenido un alcance en la universidad, comunidad y en las familias de los mismos estudiantes, una vez pasa la actividad académica el documento y los planes contenidos en él, se convierten en un instrumento de asistencia técnica en campo, puesto que orienta lo que el productor beneficiado con el estudio puede hacer para mejorar las condiciones de sus suelos, de las áreas de bosque y de la ganadería, que son el sustento diario de las familias en campo.
Hasta el momento se han atendido con asistencia técnica en las comunidades: San Miguelito, San Esteban, La Libertad, Santo Domingo (registradas las experiencias en video), La Batea, Las Praderas, El Guarumo, El Areno, Las Pavas, Cerro Blanco, Sanjón Hondo, Cedro Macho, Tamagas, entre otras, y la Estación Biológica de la facultad (los datos obtenidos han sido utilizados con fines investigativos y aporte al productor).

"La clase está súper, ahí es cuestión de nosotros poner de nuestra parte, en lo particular yo me propuse salir bien" (J. Lazo, comunicación personal)

En esta experiencia, era necesario que los estudiantes asimilaran los contenidos teóricos de la manera más rápida posible. Es por ello que se hizo énfasis en presentar un documento centrado en los elementos prácticos que reforzaban inmediatamente lo leído y analizado. Se utilizó como recurso la participación individual y se consensuaba en plenaria, a lo inmediato de terminar cada unidad se procedió a aplicar una prueba de selección múltiple con 10 ítems que se coevaluaron y discutieron sus resultados.

"El material brindado es bueno. A pesar que se recibe en cortos periodos, se trata de abarcar lo más importante para nosotros como estudiantes podamos comprender de la manera más lógica, precisa y concisa el material" (J. Piamonte, comunicación personal)

Fue necesario presentar videos previos a realizar las clases demostrativas (los videos fueros enviados por WhatsApp, para agilizar la descarga con formatos de fácil accesibilidad), para que realizaran bien la etapa de campo practicando una y otra vez hasta lograr comprender los numerosos procedimientos. Apoyarlos en los análisis de laboratorio fue un proceso intenso debido a la cantidad de estudiantes y a las condiciones de campo, se empleó tiempo adicional para realizar estas actividades.

La elaboración del reporte final lleva trabajo en equipo e independiente, los estudiantes presentaron un poco de dificultad a la hora de redacción de informes, es ahí donde la guía y el liderazgo del docente a través del acompañamiento permanente es pertinente en esta etapa de revisión. 
Como se sabe, la primera tarea del liderazgo es abrir el horizonte de posibilidades a las personas (enseñar a ver los problemas en clave de oportunidades), solo desde ahí se puede cambiar y orquestar un estado de ánimo positivo al iluminar el camino de lo que pueden lograr juntas (Casco, 2020). En este caso, elevar la calidad de los informes presentados en la evaluación final, que al finalizar todos se convierten en eventos de Extensión Universitaria devolviendo de esa manera producto a la sociedad.

\section{"Los conocimientos adquiridos en la clase de suelos han servido en años posteriores, porque he podido poner en prácticas al- gunos conocimientos". (V. Villegas, comu- nicación personal)}

A pesar de lo acelerado e intenso del curso, se observó una buena retentiva o asimilación de los contenidos, es notable que la disposición de los grupos fue notablemente positiva. La presión a la que fueron sometidos dio como resultado una calificación según la aplicación de conocimientos técnicos. Se tomó en cuenta el aporte de cada individuo y el trabajo en equipo.

\section{"Ha sido de gran ayuda ya que el conoci- miento adquirido nos beneficia a saber trabajar la tierra sin degradarla y el uso que se le puede dar al suelo que encon- tremos, el conocimiento ha servido para comprender las clases que hemos visto de manera posterior" (M. Fernández, comu- nicación personal)}

A través del tiempo, han surgido grandes variantes en el desarrollo de las asignaturas, pues la dinámica de los estudiantes ha presentado alternativas completamente diferentes, un resultado que no presenta variantes en ningún grupo es la lectura comprensiva de los documentos de la clase. Al estudiante le cuesta apropiarse del hábito de la lectura, ya sea esta de manera digital o en papel, por lo general se obliga a leer y ajustar los tiempos es importante para poder suplir esta acción, que se convierte en prioridad para mejorar esta debilidad que arrastran los estudiantes desde la escuela. En ese sentido, se ha utilizado la aplicación del Classroom de Google, no obstante, dicha plataforma causa cierta incomodidad en algunos estudiantes, pues presentan algunas fallas en cuanto a la conectividad, además de enfrentar problemas visuales al leer en formato digital. Por esta razón se imprimieron los documentos para evitar este tipo de dificultad.

Las tecnologías se deben aprovechar en nuestros tiempos, hacer ver a los alumnos que las redes sociales se pueden aprovechar, motivar el hábito de lectura, cada día el alumno ya no quiere ver un libro, un artículo, un documento; solo quiere estar conectado al mundo por medio de aparatos electrónicos, pero para lograr un proceso práctico es necesario leer, no importa en qué. Sumado a ello, el docente se muestra reacio ante el uso eficiente de los medios tecnológicos. Los centros de formación superior deben enfocarse en ello, propiciar la formación continua a todos los niveles para mejorar la calidad de la enseñanza.

\section{"La calidad de los documentos es buena} porque se explica bien lo que se va a realizar" (L. Gonzáles, comunicación personal)

Al momento de abordar este tipo de asignaturas prácticas, al docente le faltan recursos básicos para el desarrollo satisfactorio de la clase. El programa orientado plasma en sus detalles la utilización de instrumentos especializados con los que no cuenta la universidad, sin embargo, en las giras de campo los estudiantes logran conocer algunos de ellos. A su vez el docente se esfuerza por efectuar la clase aportando algunos de ellos, ya que, si no hay, el proceso se vuelve más teórico.

Las estrategias aplicadas es 50\% teoría y 50\% practicas, para todas las clases cada tema está relacionado a efectuar una actividad en campo. Llevan un diseño secuencial, por tanto, el estudiante debe apropiarse de la dinámica cada semana y cada semestre (la primera clase contiene los conocimientos básicos para entender la segunda parte de manera más eficiente).

$$
\begin{gathered}
\text { "Profe, jes lo máximo! Deberíamos prac- } \\
\text { ticar más" (Y. Centeno, comunicación } \\
\text { personal) }
\end{gathered}
$$


Para L. Luzuriaga, el carácter activo de la educación se revela en la concepción del método educativo de Dewey, siendo sus rasgos principales los siguientes:

- Que el alumno tenga una situación de experiencia directa, es decir, como una actividad continua en la que esté interesado por su propia cuenta

- Que se plantee un problema autentico dentro de esta situación, como un estímulo para el pensamiento.

- Que posea la información y haga las observaciones necesarias para tratarla.

- Que las soluciones se le ocurran a él, lo cual le hará responsable de que se desarrollen de un modo ordenado.

- Que tenga oportunidades y ocasiones para comprobar sus ideas por sus aplicaciones, aclarando así su significación y su descubrimiento por sí mismo, su validez.

Muchas de las actividades mencionadas, las realizan en la Reserva Silvestre Estación Biológica "Francisco Guzmán Pasos" en donde se cumplen múltiples funciones, ya que este lugar de estudio es considerado un laboratorio vivo, desarrollando parcelas experimentales, cuido de animales, monitoreo de flora y fauna, entre otros. El estudiante tiene a disposición, en este espacio, elementos del medio biótico para desarrollar prácticas que fortalecen los conocimientos teóricos adquiridos. De igual manera, se contribuye dejando investigaciones y proyectos de Extensión Universitaria como: diagnósticos comunitarios, embellecimiento de jardines escolares, charlas ambientales a niños, escuelas de campo con pequeños productores, por mencionar algunos.

"La clase estuvo bien entretenida y aprendí mucho sobre poda, el objetivo de esta y para que se hace, y la importancia de los bosques". (R. Salazar, comunicación personal)

Es importante mencionar, que parte de las clases incluyen la invitación de expertos en los diversos temas, profesionales que están vinculados con el quehacer institucional del Gabinete de la Producción, donde se tiene la oportunidad de que ellos compartan parte de las realidades con los estudiantes, son espacios complementarios que se aprovechan al máximo para estrechar relaciones con los actores en el territorio.

\section{"La clase es bonita e interesante, y genera mucho aprendizaje. Las actividades de campo que se realizan son buenas porque así se aprende más". (L. Borges, comunica- ción personal)}

Estas actividades complementarias ha permitido caracterizar las habilidades, conocimientos previos y disposición de trabajo en los estudiantes, por lo general han mostrado una buena disposición pero en algunas ocasiones carentes de entusiasmo. Quizás los tiempos de crisis que estamos viviendo en la actualidad han influido en ese sentimiento de angustia, costando un poco sacar a los jóvenes de ese estado mental, también han tenido sesiones especiales que promueven el trabajo en equipo, autoestima y autoconcepto, con ello se han logrado mitigar las situaciones negativas y hacer más llevadera las crisis.

"La clase no se hizo difícil, pero tampoco fácil” (M. Sequeira, comunicación personal)

Contando una de las experiencias, se les llevó a una gira de campo al Centro Genético Forestal de Nicaragua, donde vieron procesos de recolección, selección, secado, empacado y distribución de semillas. Observación de las parcelas experimentales con las diversas especies que se promueven en el país, y realizaron una práctica de escalamiento de árbol, con sus debidas medidas de seguridad.

\section{"No sabía que escalar un árbol es una tarea tan peligrosa y que requiere de especialistas" (D. Díaz, comunicación personal)}

Con todo esto, queda claro que la preparación de profesionales en carreras afines al campo agropecuario como menciona Casco (2018), fortalecerá a que el mundo rural siga cumpliendo sus funciones tradicionales relacionadas con la conservación del medio, producción de alimentos, espacio de solaz y disfrute...; pero además de ese rol, tiene la posibilidad de generar, promover y liderar nuevas actividades 
relacionadas con las tendencias emergentes de la economía y de la idiosincrasia de los pueblos. La secuencia a edafología, forestal la articulación de agrostología, fisiología vegetal, silvicultura y dasometría, para luego ver sistemas de producción animal, pretende que el alumno de manera clara logre asimilar su interrelación.

El suelo provee siempre y cuando se respete, poder saber lo que contiene de ahí su mayor importancia y poder cuidar, que las plantas se desarrollan a través de él y son alimentos para todos y para el mismo suelo y que los animales también proveen vida a través de sus deyecciones que se incorporan al suelo y nutren las plantas, pero también a través de sus subproductos.

\section{"Buen sistema de evaluación, el docente tiene disponibilidad de atender dudas, aporta y da aclaraciones" (D. Báez, comu- nicación personal)}

En el desarrollo de clases prácticas, es muy importante contar con los recursos adecuados, acá se refiere a un medio, instrumento o herramienta que es necesario para poder completar una demostración práctica. Nuestras universidades públicas hacen un gran esfuerzo en adecuarse a lo que recibe del Presupuesto General de la República, que a su vez se ve afectado por los sucesos sociopolíticos que surgen dirigidos o por eventualidades.

De ahí se debe cubrir todas las demandas y eso limita el accionar. Acá el docente y alumno se ven obligados a emprender, poder suplir con recursos locales las necesidades, además, muchas veces el docente se ven en la necesidad de adquirir sus herramientas para poder tener mayor desempeño en sus clases.

Una vez que pasas del aula a la Unidad de Producción, llegas con el productor y encuentras que este ejerce todas sus actividades agropecuarias porque así se las enseñaron sus antepasados, encuentras otra barrera: el poder convencer al productor por medio de hacer y aprender, pero que, si el alumno no es capaz de lograr esto, pues el éxito no se dará.

Acá es importante la participación del docente, que sea capaz de ganar la confianza del productor para que se manipule un cultivo, un pasto, un lote de animales, una especie de interés y lo más importante que lo que te facilita no sea alterado o dañado por el alumno, sino que se mejore. El productor identifica inmediatamente cuando el alumno o docente no está haciendo algo bien y lo hará saber.

La importancia, entonces, es la formación del recurso humano que imparte una clase práctica. Muchas veces los errores que se cometen es que un docente se convierte en súper docente, pudiendo impartir muchas asignaturas que no están dentro de su formación, o bien, que no los motiva mucho, esto suele suceder en países en vías de desarrollo en donde se delegan asignaturas a profesionales que todavía no reúnen las cualidades para eso. De ahí, pues, el impacto que llega a tener en el alumno poder realizar una clase práctica sea de campo, laboratorio, en aulas de clases o cualquier otra.

\section{"El profe nos exige más allá de nuestras fuerzas, eso nos motiva a mejorar" $(0$. Ojeda, comunicación personal)}

De pronto toca que en tu plan de clases se debe realizar un diagnóstico de gestación a través de la palpación transrectal, pero para ello, el docente debe estar previamente formado y debe ser capaz de transmitir al alumno eso por medio de ilustraciones, practicas con órganos reales, por medio de animales vivos y repetir y repetir, pero sucede que el plan de clases solo te dice que son algunos encuentros, en esto y si el docente no está realmente adiestrado, los resultados que se buscan no serán completados. Esto puede suceder también cuando realizamos diferentes técnicas de injertación en cultivos o balance de raciones para diferentes especies animales.

El profesional debe estar formado para lo que debe trasmitir y debe saberlo trasmitir. Te encuentras entonces que un docente que está impartiendo una asignatura resulta que debe realizar una visita práctica guiada y no maneja lo que se debe hacer, esto inmediatamente lo identifica el alumno y se forma de manera somera su idea sobre que está haciendo y el interés de ese docente que no logra motivar.
"La clase no es difícil porque la persona sabe dar la asignatura, las actividades estaban para que todos las hiciéramos"
(K. Lumbí, comunicación personal) 
"El mundo no es. El mundo está siendo. Como subjetividad curiosa, inteligente, interviniente en la objetividad con la que dialécticamente me relaciono, mi papel en el mundo no es sólo de quien constata lo que ocurre, sino también de quien interviene como sujeto de lo que va a ocurrir. No soy un mero objeto de la historia sino, igualmente, su sujeto" Paulo Freire.

\section{Lecciones aprendidas}

A lo largo de los años, estas experiencias nos han dejado grandes aprendizajes, como docentes tenemos el compromiso de continuar abiertos a sentir y experimentar el aprendizaje colaborativo pues ¡uno, no es ninguno! y la colaboración es la base para llegar hacia metas concretas. Esa unión no es pensar al 100\% lo mismo que el otro, sino estar cierto de qué piensa la otra persona y de lo que piensas tú.

$Y$ en ese sentido, los éxitos y fracasos han dependido del pensamiento constructivista, de sueños en donde se ve un aporte al desarrollo, por muy insignificante que parezca. Estas habilidades, caracterizan y sirven de indicadores de un desempeño individual y colectivo de estudiantes y docentes, en el proceso de apropiación del conocimiento como acción que sin lugar a dudas constituye una vía básica para el aprendizaje.

Para los centros públicos de educación superior que tienen presencia en los territorios rurales deben consolidar sus metodologías de formación. El poder formar a un hijo de un productor rural que dé respuesta en un determinado territorio generará un impacto positivo que conllevará a mejorar la productividad y abonar a la consolidación económica de una región. A veces se cree que el alumno del sector rural asimila más lento el aprendizaje en comparación del alumno del sector urbano, mientras que el alumno del sector rural tiene más experiencia en campo que el del sector urbano. Para no cometer ni caer en estos errores, el docente debe ser capaz de identificar y poder nivelar o aprovechar cualquier indicio. Ciertamente el docente siempre enriquece su formación sabiendo asimilarlo y trasmitirlo.

Hay algunos grupos en donde los estudiantes hacen las pruebas en sus propias fincas, siendo los beneficiarios ellos mismos y sus familias. Esta acción repercute en cómo ven los estudiantes a sus padres (en la mayoría de las ocasiones los chavalos tienen temor de acercarse a sus padres), y a su vez el cómo los padres ven los avances en la formación de sus hijos, los ven como el hijo que va a cometer errores, pero pronto van cambiando su perspectiva y poco a poco logran verlos como los futuros profesionales al servicio de sus propios beneficios.

Las asignaturas vistas de manera secuencial facilitan a los estudiantes poder comprender como se articulan los diferentes medios y recursos que provee una unidad de producción, sin perjuicio de su sobreexplotación. Al tener en Nicaragua diferencias en los microclimas, cada región es única, tienen sus propias riquezas y eso ayuda a encontrar diferentes actividades agropecuarias que se incorporan en la producción nacional, por ende, un sinfín de recursos que pueden ser utilizados en los procesos educativos. La identificación de los recursos locales como parte del emprendimiento puede ser la motivación necesaria para lograr satisfacer una demanda y su transformación para lograr establecer medios de formación.

Se debe aprender a dar el valor adecuado a los recursos naturales con los que se cuenta, de ellos vivimos, nos alimentamos, nos superamos, al final a ellos regresamos. Los recursos naturales de los países en desarrollo son el medio más preciado para su desarrollo, todo esto con el objetivo de aportar a la mejora de la calidad de vida en el campo agropecuario, y dar una parte de las respuestas a las necesidades básicas que tienen que ver con la educación y el bienestar, la producción sostenible y la protección de la madre tierra en nuestros territorios.

A través de los años, la experiencia se vuelve interesante, puesto que, la aplicación de metodologías basadas en el aprendizaje autónomo, con todos y en todos los momentos no da resultados positivos todo el tiempo, lo cual, dependerá en gran medida del entusiasmo impreso desde el inicio y si los estudiantes tienen actitudes pasivas o activas. Para esos casos de estudiantes pasivos, aunque se les presente la clase y las actividades de manera dinámica, la respuesta de los jóvenes llega a ser por cumplimiento y enfocados en entregar un producto de mediana calidad y obtener una calificación. Asunto que de alguna manera se ha logrado mitigar a través de reenfocar las clases en el momento, orientándolas al desarrollo de otras habilidades que existen en las mentes de los jóvenes, logrando así un poco más de interacción.

Para facilitar la labor docente, debe tener un seguimiento de los procedimientos, revisiones y discusión entre el colectivo, y una vez que se van 
teniendo experiencias en una asignatura se puedan colectivizar algunos contenidos, matrices que sean fáciles de seguir en sus procedimientos. Estas lecciones aprendidas son las que se han alcanzado, pues hay que pasar por la experiencia de las diferentes asignaturas que desarrollas para poder compartir un poco y hacer de la docencia un fenómeno motivador.

\section{Conclusiones}

Cada uno construye su propia historia a partir de experiencias que son únicas e irrepetibles. En campo suceden cosas que no cuenta con un libro o un manual. Es ahí, donde el docente debe valorar, atesorar, disfrutar esos momentos a lo largo de los años, mantener esa "primera impresión", "el perderla es trágico", el volver a ella es renovador de fuerzas y ánimos de saber qué haces algo que está funcionando (aunque a veces no, de ahí se hace una reflexión desde el exterior al interior). A unos les funciona por cumplimiento, a otros por necesidad y a otros porque se apasionan. Uno de los hitos de mayor relevancia en esta experiencia, es el haber roto esos esquemas de la relación familia - hijos, pues los padres al dejar a sus hijos realizar estos experimentos en sus propios lugares, es un paso muy importante en la vida de los jóvenes.

\section{"Estamos poniendo en práctica todo lo de las clases, para la poda hemos buscado unos serruchos que se pueden enganchar en tubos porque son más fuertes" (B. Scot- to, comunicación personal)}

De igual manera, se logra apreciar el liderazgo del maestro quien de manera sorpresiva, impulsa todas estas interacciones al dejar que los estudiantes hagan estas actividades de manera independiente, esto lo consideramos como uno de los impactos sociales, familiares y productivos más relevantes de la experiencia educativa.

Otro mensaje importante, es que instamos a todos los individuos que utilicen o gestionen el suelo, el agua, los bosques, deben actuar como administradores de este. A fin de velar por la gestión sostenible de este recurso natural esencial para salvaguardarlo en beneficio de las generaciones futuras. Por tanto, los recursos naturales bien cuidados son un aliado crucial para asegurar la seguridad alimentaria y nutricional de nuestros pueblos, así como ser un recurso de aprendizaje infinito, dando cumplimiento a los Objetivos de Desarrollo Sostenible.

El materializar los productos creados por los estudiantes en las diferentes asignaturas y años lectivos, ha permitido la dinamización de los ciclos de aprendizajes que trascienden a sus vidas, y los docentes somos privilegiados observadores.

\section{Referencias bibliográficas}

- Ávila, M.L., \& Cortés, M.J.(2017). La Sistematización de experiencias educativas. Una experiencia con docentes universitarios. European Scientific Journal, 137-153. doi:10.19044/esj.2017.v13n4p137

- Bartolomé, P. M. (2017). Diversidad educativa ¿Un potencial desconocido? Revista de Investigación Educativa, 15-33.

- Casco, J. C. (2 de Julio de 2018). http://www. emprendedorex.com/. Obtenido de https://cutt.ly/ orHrMSW

- Casco, J. C. (3 de Febrero de 2020). http://www. emprendedorex.com/. Obtenido de https://cutt.ly/ zrHwjnp

- CEPES. (2002). Tendencias Pedagógicas en la Realidad Educativa Actual . Bolivia: Editorial Universitaria, Universidad Juan Misael Sarancho.

- Freire, P. (1981). Educação e Mudança. Río de Janeiro.: Paz e Terra.

- Gobierno de Nicaragua. (2017). El paradigma educativo centrado en el aprendizaje, Nicaragua un nuevo modelo educativo basado en el aprendizaje. Managua: Asistencia técnica: Emprendedorex.

- ICCP. (2002). COMPENDIO DE PEDAGOGIA . Cuba: Instituto Central de Ciencias Pedagógicas (ICCP).

- López Díaz, R. A. (2017). Estrategias de enseñanza creativa : investigaciones sobre la creatividad en el aula. Bogotá: CLACSO.

- Melgar, M. F., \& Donolo, D. S. (2011). Salir del aula... Aprender de otros contextos: Patrimonio. Revista Eureka sobre Enseñanza y Divulgación de las Ciencias, 323-333. 
- $\quad$ Melgar, M. F., Elisondo, R. C., \& Donolo, D. S. (2016). El poder educativo de lo inesperado. Estudio de experiencias innovadoras en la universidad. Cuadernos de Investigación Educativa, 31-47.

- Moore, F. P. (2018). Experiencias educativas innovadoras en carreras e grado: El Casodel Instituto Tecnológico De Buenos Aires. Revista Argentina de Educación Superior, 87-97.

- UNESCO. (2015a). Replantear la Educación. Francia: Organización de las Naciones Unidas para la Educación, la Ciencia y la Cultura. Obtenido de http://unesdoc.unesco.org/

- UNESCO. (2015b). Declaración de Incheón 2030 y Marco de Acción . Francia: UNESCO. Obtenido de http://www.unesco.org

- Zapata, D. (2014). ACTIVIDADES DE LAS GUÍAS DE APRENDIZAJE DE ESCUELA NUEVA. Universidad de Antioquia , 64-66.

\section{ANEXOS}

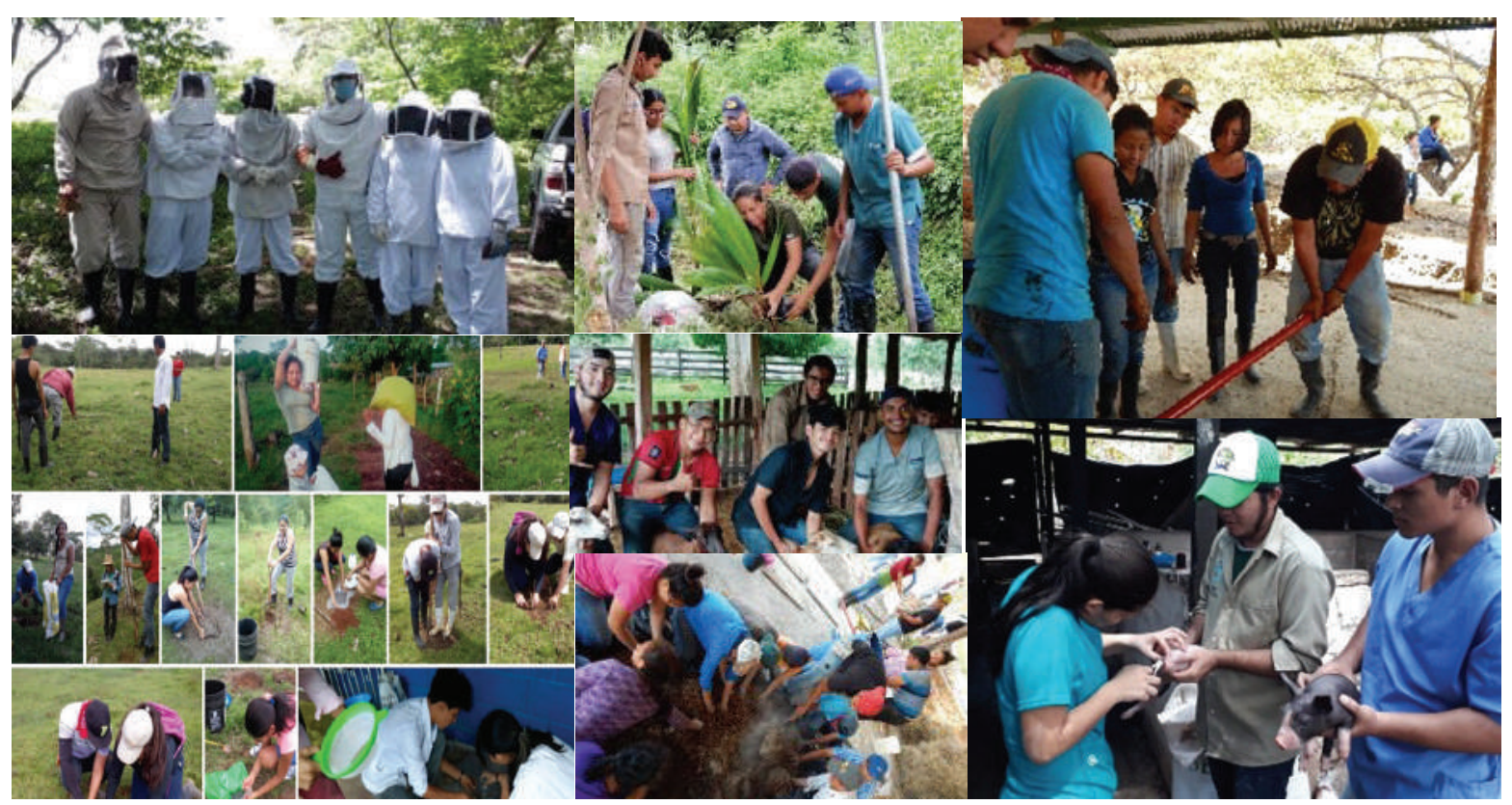

